\title{
Heat shock transcriptional factors in Malus domestica: identification, classification and expression analysis
}

\author{
Filomena Giorno ${ }^{1,2^{*}}$, Gea Guerriero', Sanja Baric ${ }^{1}$ and Celestina Mariani ${ }^{2}$
}

\begin{abstract}
Background: Heat shock transcriptional factors (Hsfs) play a crucial role in plant responses to biotic and abiotic stress conditions and in plant growth and development. Apple (Malus domestica Borkh) is an economically important fruit tree whose genome has been fully sequenced. So far, no detailed characterization of the Hsf gene family is available for this crop plant.

Results: A genome-wide analysis was carried out in Malus domestica to identify heat shock transcriptional factor (Hsf) genes, named MdHsfs. Twenty five MdHsfs were identified and classified in three main groups (class A, B and C) according to the structural characteristics and to the phylogenetic comparison with Arabidopsis thaliana and Populus trichocarpa. Chromosomal duplications were analyzed and segmental duplications were shown to have occurred more frequently in the expansion of Hsf genes in the apple genome. Furthermore, MdHsfs transcripts were detected in several apple organs, and expression changes were observed by quantitative real-time PCR (qRT-PCR) analysis in developing flowers and fruits as well as in leaves, harvested from trees grown in the field and exposed to the naturally increased temperatures.
\end{abstract}

Conclusions: The apple genome comprises 25 full length Hsf genes. The data obtained from this investigation contribute to a better understanding of the complexity of the Hsf gene family in apple, and provide the basis for further studies to dissect Hsf function during development as well as in response to environmental stimuli.

Keywords: Hsf, Malus domestica, Gene expression, High temperature, Apple fruit/ flower

\section{Background}

Trees are sessile organisms with long lifespans that regularly experience climatic fluctuations in their native environment. Therefore, survival and reproduction is dependent upon an array of protective mechanisms that involve the activation of a wide range of transcriptional factors, and their products are considered to play a central role in response to extreme physiological conditions. There is evidence that members of the heat shock transcriptional factor (Hsf) family are important regulators in sensing and signaling of different environmental stresses [1]. Similarly to many other transcription factors, the Hsfs have a modular structure containing signature

\footnotetext{
* Correspondence: mgiorno@science.ru.nl

'Research Centre for Agriculture and Forestry Laimburg, Laimburg 6, Auer/ Ora, BZ, 39040, Italy

${ }^{2}$ Department of Molecular Plant Physiology, Radboud University Nijmegen, Heyendaalseweg 135, Nijmegen 6525 AJ, The Netherlands
}

domains structurally and functionally conserved throughout the eukaryotic kingdom. A common core structure in the Hsfs is composed of an $\mathrm{N}$-terminal DNA binding domain (DBD), characterized by a central helix-turn-helix motif that specifically binds to the heat shock elements (HSE) in the target promoters, and an adjacent bipartite oligomerization domain (HR-A/B) composed of hydrophobic heptad repeats [2]. Hsf trimerization via the formation of a triple stranded alphahelical coiled-coil is a prerequisite for high affinity DNA binding and, subsequently, for transcriptional activity. Other Hsf functional modules include clusters of basic amino acids essential for nuclear import (NLS), leucinerich export sequences important for nuclear export (NES), and a less conserved C-terminal activator domain (CTAD) rich in aromatic, hydrophobic and acidic amino acids, the so-called AHA motifs $[2,3]$. 
In contrast to Saccharomyces cerevisiae, Caenorhabditis elegans, and Drosophila melanogaster, that each possesses only a single Hsf gene, plant genomes contain large numbers of $H s f$ genes, up to $52[1,4,5]$. Based on structural characteristics and phylogenetic comparisons, plant Hsfs are grouped into classes A, B and C [2,6]. All class $\mathrm{A}$ and $\mathrm{C}$ Hsfs have an extended $\mathrm{HR}-\mathrm{A} / \mathrm{B}$ region due to the insertion of 21 (Class A) or seven (class $C$ ) amino acid residues between $A$ and $B$ parts of the HR$\mathrm{A} / \mathrm{B}$ region. On the contrary, in class $\mathrm{B}$ Hsfs, the HR-A/ $B$ region does not contain insertions. In addition, sequence comparisons and structural analyses indicate that the combination of a AHA motif with an adjacent nuclear export signal NES represents a peculiar signature domain for many plant class A Hsfs [6,7].

After the release of the whole genomic sequences of several plant organisms, including rice (Oryza sativa), maize (Zea mays), poplar (Populus trichocarpa), medicago (Medicago truncatula), tomato (Solanum lycopersicon), the Hsfs family was analyzed extensively, both to place each member in an organized nomenclature system and to provide maps of their expression [7-10].

Recently, the full genome sequence of the domesticated apple (Malus domestica Borkh) has been published [11]. This provides a useful genomic tool to study this economically important fruit crop. As transcriptional factors, Hsfs are involved in different aspects of plant life including tolerance to biotic/abiotic stresses and developmental processes [12-14]. Therefore, this gene family represents an important group of transcriptional factors to investigate and to characterize. Genome scale analyses of the transcriptional response during development and to environmental stimuli require a precise and complete annotation of genes in order to provide reliable and exhaustive data. Therefore, the aim of this study was to annotate the full length $H s f$ genes in apple, and to analyze their expression profiles by quantitative real time PCR (qRT-PCR) in different organs/tissues from plants grown in the field and exposed to natural environmental conditions. The results of this work provide a foundation to better understand the functional structure and genomic organization of the Hsf gene family in apple, and will be undoubtedly useful in future gene cloning and functional studies.

\section{Results}

Identification, classification and duplication of Hsf genes in the Malus domestica genome

CDS sequences corresponding to putative $H s f$ genes from Malus domestica (MdHsfs) were searched in the Apple Genome v1.0 [15]. As a result, 36 genes encoding for putative MdHsfs proteins were identified. All candidate MdHsf proteins were surveyed, and incomplete sequences for the DBD domain and for the remaining functional domains were removed. This resulted in the selection of twenty five complete sequences. These $M d H s f$ genes were distributed on 12 of the 17 apple chromosomes with the largest number, comprised of six Hsf genes, detected on chromosome 15 (Table 1). According to the multiple sequence alignment of the DBD and HR-A/B region, 16 genes were determined to be Class A, seven genes were identified as Class B and two were classified as Class $\mathrm{C}$.

Gene duplication events have been indicated as an important mechanism in the evolution of plant genomes [16]. Therefore, duplications of $M d H s f s$ were also analyzed. As shown in Figure 1, a total of 12 duplicated gene pairs of $M d H s f s$ were identified, including 11 segmental duplication events between chromosomes (e.g. $M d H s f C 1 a$ and $M d H s f C 1 b)$ as well as one tandem duplication event within the same chromosome, e.g. $M d H s f A 3 c$ and $M d H s f A 3 b . M d H s f A 3 c$ was the only $H s f$ involved in both duplication events, as it was duplicated with $M d H s f A 3 b$ in tandem on chromosome 14 and also segmentally duplicated with MdHsfA3a on chromosome 12 .

Table 1 List of Hsfs genes in the Malus domestica genome

\begin{tabular}{|c|c|c|c|c|c|}
\hline Gene name & Chromc & somal localization & Size $(\mathbf{a a})$ & $\mathrm{MW}(\mathrm{kDa})$ & $\mathrm{pl}$ \\
\hline MdHsfAla & Chr6 & MDP0000517644 & 540 & 59.37 & 4.76 \\
\hline 16 & $C$ & $\Lambda$ & 5 & 4 & 96 \\
\hline MdHsfATC & Chr13 & MDP0000232623 & 550 & 60.07 & 6.01 \\
\hline MdHsfAld & Chr16 & MDP0000259645 & 580 & 64.34 & 5.04 \\
\hline MdHsfAZa & Chr8 & MDP0000489886 & 380 & 42.42 & 4.73 \\
\hline$M d H s f A 2 b$ & Chr15 & $\mathrm{MDF}$ & 3 & 4 & 4.63 \\
\hline MdHsfA3a & Chr12 & MDP0000131346 & 516 & 56.26 & 4.18 \\
\hline MdHsfA3b & Chr14 & MDP0000606400 & 455 & 50.37 & 6.43 \\
\hline$M d H$ & 14 & $\mathrm{ML}$ & 5 & 64.34 & 4.89 \\
\hline MdHsfA4a & Chr5 & MDP0000155849 & 420 & 47.23 & 5.62 \\
\hline MdHsfA5a & hrg & MDP0000301101 & 483 & 33.01 & 5.08 \\
\hline MdHsfA5b & Chr15 & MDP0000613011 & 482 & 54.19 & 5.48 \\
\hline a & C & $\mathrm{ML}$ & 4 & 46.89 & 4.55 \\
\hline MdHsfABb & Chr13 & MDP0000172376 & 411 & 44.86 & 5.10 \\
\hline MdHsfA9a & Chr2 & MDP0000194672 & 713 & 75.89 & 6.86 \\
\hline MdHsfAgb & Chr15 & MDP0000319456 & 482 & 53.29 & 4.86 \\
\hline MdHsfBla & Chr2 & MDP0000527802 & 294 & 32.26 & 8.76 \\
\hline$M d H s f B 1 b$ & Chr15 & MDP0000578396 & 23 & 20 & 4.67 \\
\hline MdHsfB2a & Chr1 & MDP0000155667 & 276 & 30.97 & 5.96 \\
\hline$M d F$ & Chr12 & $\mathrm{MD}$ & 2 & 27.77 & 7.22 \\
\hline$M d H s f B 3 b$ & Chr14 & MDP0000202716 & 243 & 27.82 & 7.82 \\
\hline$M d H s f B 4 a$ & Chr8 & MDP0000209135 & 381 & 42.85 & 7.62 \\
\hline$M d H s f B 4 b$ & Chr15 & MDP0000129357 & 383 & 43.19 & 7.64 \\
\hline MdHsfCla & Chr2 & MDP0000230456 & 324 & 36.25 & 6.27 \\
\hline MdHsfClb & Chr15 & MDP0000320827 & 344 & 38.36 & 5.02 \\
\hline
\end{tabular}

MW: molecular weight; pl: Isoelectric point. 


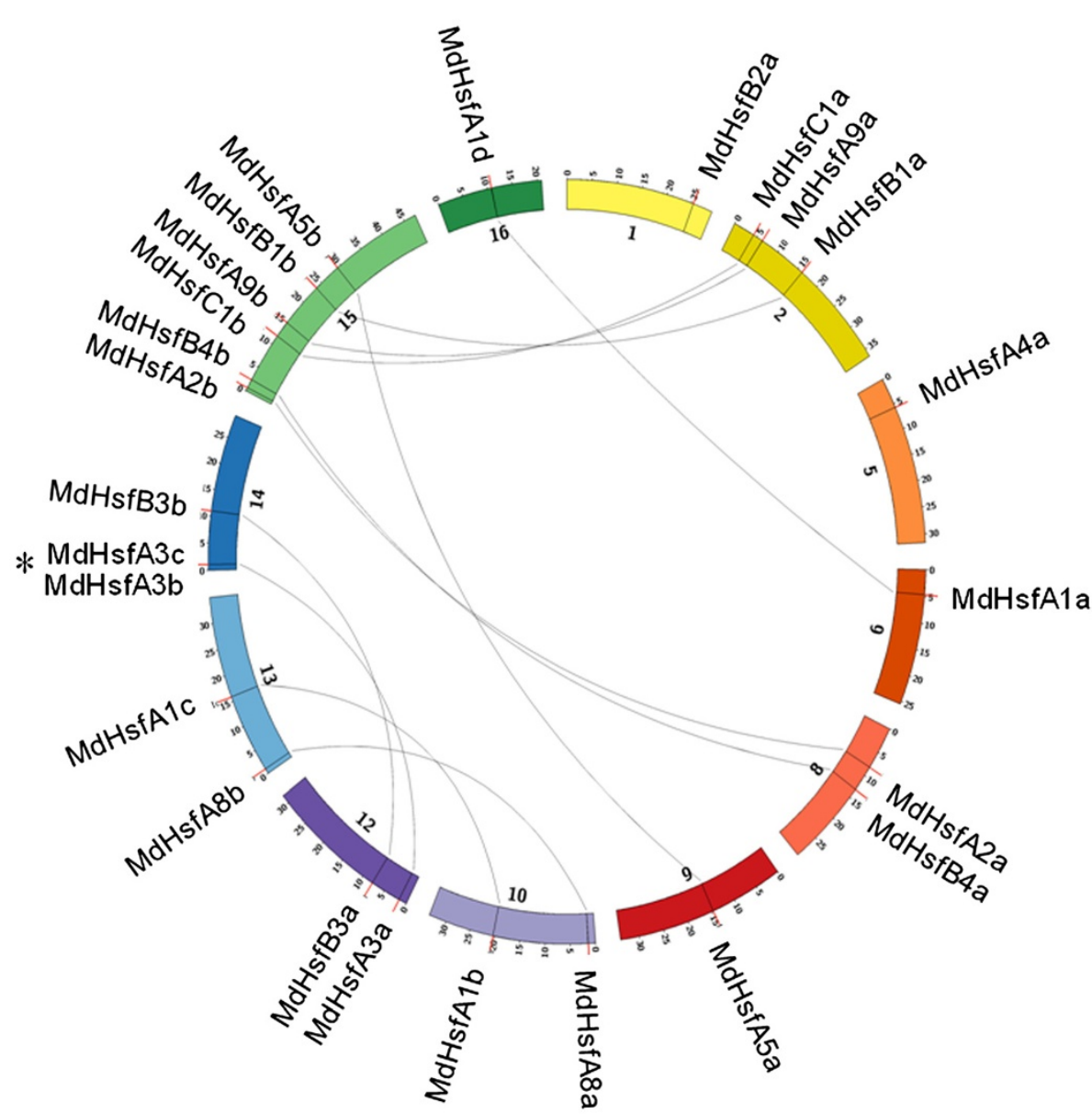

Figure 1 Localization and duplication of the Hsf genes in the apple genome. Circular visualization of the $25 \mathrm{Hsfs}$ mapped on the different chromosomes in the apple genome was obtained using the Circos software. Picture shows only the chromosomes containing MdHsf genes, and chromosome number is indicated on the inner side. Segmental duplications were joined by the lines, while the tandem duplication of MdHsfA3b and MdHSfA3C is indicated by an asterisk.

Analysis of conserved domains in the apple Hsf proteins Prediction of the typical signature domains present in the MdHsfs protein sequences was carried out by comparing the identified apple Hsfs to those of homologous, well characterized proteins of model plants such as tomato or Arabidopsis [2,6,7]. Table 2 lists five conserved motifs that were identified by sequence alignment, and their positions in the protein sequences. All the MdHsfs showed the presence of the highly conserved DBD domain in the $\mathrm{N}$-terminal region, consisting of a threehelical bundle ( $\mathrm{H} 1, \mathrm{H} 2$ and $\mathrm{H} 3)$ and a four-stranded antiparallel $\beta$-sheet. The length of the DBD motif was quite variable with the smaller size observed for MdHsfB1b. The presence of the coiled-coil structure characteristic of leucine-zipper type protein interaction domains, which is a property of the HR-A/B region, was instead predicted in all MdHsfs proteins by using MARCOIL tool. Furthermore, the majority of the MdHsfs showed the presence of NES and NLS domains which were described to be essential for shuttling Hsfs between nucleus and cytoplasm [7]. Additional sequence comparison allowed the identification of AHA motifs in the center of the C-terminal activation domains, as it is expected in the A-type Hsfs. By contrast, these domains were not identified in the B and C-type MdHsfs.

A second approach was used to identify and to verify domain prediction in the MdHsf proteins, by using the MEME motif search tool. Thirty corresponding consensus motifs were detected (Figure 2; Table 3). The majority of MdHsfs displayed the presence of the motifs 1, 2, $3,4,5$ which correspond to highly conserved regions including the $\mathrm{DBD}$ and $\mathrm{HR}-\mathrm{A} / \mathrm{B}$ region domains. In addition, the inspection of motif distribution revealed that some of them were only present in specific classes of the MdHsf family. For example, motif 10 was representative of A-type Hsf members such as MdHsfA1a-A1d, MdHsfA4a, MdHsfA8a, and it contained the signature domains corresponding to NES sequence. Similarly, 
Table 2 Functional motifs of apple Hsfs

\begin{tabular}{|c|c|c|c|c|c|}
\hline Gene name & DBD & HR-A/B & NLS & NES & AHA \\
\hline MdHsfAla & 39-132 & $154-220$ & (242) NKKRRLKK & (502) MDNLTEKMG & AHA (454) DIEAFLKDWDD \\
\hline MdHsfA1b & 17-152 & $174-240$ & (264) NKKRRLPR & (529) MNHITEQM & AHA (482) DIFWEQFLTAS \\
\hline MdHsfA1c & $16-156$ & $173-243$ & (268) NKKRRLPR & (533) MNHITEQMQ & AHA (486) DIFWEQFLTAS \\
\hline MdHsfAld & 103-196 & $217-284$ & (307) NKKRRLKR & (563) MDNLTEKMG & AHA (516) DIEAFLKDWDD \\
\hline MdHsfA2a & 30-132 & $147-213$ & (228) $\mathrm{KNRK} \mathrm{X}_{7}-\mathrm{RKRR}$ & (368) LLDQMGYQ & AHA1 (318) ETIWEELWSD AHA2 (360) DWGKDLQD \\
\hline MdHsfA2b & 38-131 & $145-212$ & (227) KNR-X 6 -RKRR & (365) LVDQMGYL & AHA1 (315) ETIWEELWSD AHA2 (355) DWGEDLQD \\
\hline MdHsfA3a & 99-209 & $226-285$ & (297) KTRRKFVK & nd & $\begin{array}{l}\text { AHA1 (431) EDIWSMGFGV AHA2 (450) ELWGNPVNY AHA3 } \\
\text { (470) LDWWDIGPLQ AHA4 (486) IDKWPAHDS }\end{array}$ \\
\hline MdHsfA3b & 99-232 & $253-312$ & (328) KDIGSSRVRRKFVVK & nd & nd \\
\hline MdHsfA3C & $99-244$ & $265-324$ & (340) KDIGSSRVRRKFVVK & nd & $\begin{array}{l}\text { AHA1 (500) EDIWSMNFDV AHA2 (518) NELWGNPXNY } \\
\text { AHA3 (539) LDWWDIDPLQ AHA4 (555) INKWPAHES }\end{array}$ \\
\hline MdHsfA4a & 10-103 & $123-190$ & (208) RKRRLPR & (407) LTEQMGHL & AHA1 (252) LTFWEDTIHD AHA2 (356) DGFWEQFLTE \\
\hline MdHsfA5a & 12-105 & 116-183 & (194) RK-X ${ }_{10}-$ KKRR & (477) AETLTL & AHA (431) DVFWEQFLTE \\
\hline MdHsfa5b & 12-105 & 117-183 & (194) RK-X ${ }_{10}$-KKRR & (477) AETLTL & AHA (431) DVFWEQFLTE \\
\hline MdHsfAsa & 18-111 & 129-199 & (177) RNRLR & (389) TEQMGHL & AHA (308) DGAWEQFLLA \\
\hline MdHsfABb & 18-111 & 127-196 & (172) RLLRNR & nd & AHA (306) DGAWEQLLLG \\
\hline MdHsfagb & $139-239$ & $241-308$ & (324) KR-X - KRRR & (258) LKADQD & nd \\
\hline MdHsfagb & $139-239$ & $241-308$ & (324) KR-X - KRRR & (258) LKADQD & nd \\
\hline MdHsfB1a & $6-99$ & $142-191$ & (246) KGDEKMKGKK & nd & nd \\
\hline MdHsfBIb & $2-35$ & $78-127$ & (181) KGEEKMKGKK & (159) LDMEGG & nd \\
\hline MdHsfB2a & 22-115 & 154-197 & (167) RLRK & nd & nd \\
\hline MdHsfB3a & 19-112 & 149-194 & (223) RKRKR & (208) PKLFGVRLE & nd \\
\hline MdHsfB3b & $22-116$ & 149-194 & (179) KRKCK (223) RKRKR & (208) LKLFGVRLE & nd \\
\hline MdHsfB4a & 21-114 & $183-239$ & (325) KNTK-X $X_{9}-K K R$ & (366) LEKDDLGLQLM & nd \\
\hline$M d H s f B 4 b$ & 21-114 & $180-240$ & (327) KNTK-X $\mathrm{X}_{9}-\mathrm{KKR}$ & (368) LEKDDLGLHLM & nd \\
\hline MdHsfCla & $7-100$ & 119-171 & (195) KKRR & nd & nd \\
\hline MdHsfClb & $9-102$ & $128-180$ & ( 204) KKRR & nd & nd \\
\hline
\end{tabular}

Number in brackets indicates the position of the first amino acid present in the putative nuclear localization signal (NLS), nuclear export signal (NES) and activator (AHA) motifs in the C-terminal domains. nd, no motifs detectable by sequence similarity searches.

motif 7 containing the AHA sequence was detected in the C-terminal parts of many MdHsf proteins, belonging to the A class. Furthermore, eight A-type Hsfs members, namely MdHsfA1a-A1d, MdHsfA2a-b, MdHsfA5a-b were characterized by the presence of motif 13 which contained the NLS domains. Interestingly, all B Hsfs members exhibited motif 20, while MdHsfC1a and MdHsfC1b contained the motif 29 (Table 3).

\section{Phylogenetic analysis of apple Hsf proteins}

To investigate the evolution of Hsfs an unrooted phylogenetic tree was generated by using the 25 Malus domestica Hsfs, 28 Populus trichocarpa Hsfs (PtHsfs) and 21 Arabidopsis thaliana Hsfs (AtHsfs). Populus and Arabidopsis were chosen because their full sequence genome has been released, and Hsf members have been well characterized $[7,10]$. Moreover, the former is a tree.
Figure 3 shows the result of this analysis. Hsfs of Malus domestica, Arabidopsis thaliana and Populus trichocarpa were clearly grouped into three different clades corresponding to the main Hsf classes A, B and C. Within the A-type clade, nine distinct sub-clades were resolved, seven of which (A1, A2, A3, A4, A5, A8 and A9) comprised the apple Hsf sequences. The C-type Hsfs from the three plant species also constituted one distinct clade which appeared more closely related to the Hsf A-group. Correspondingly, the B-type Hsfs from the three plant species grouped in a separate clade. Two of the five sub-clades, B3 and B2, were paraphyletic. As expected, the duplicated Hsfs of Malus domestica clustered all together on the phylogenetic tree.

\section{In silico expression analyses of $M d H s f$ genes}

Tissue specific expression of $M d H s f s$ was investigated by counting the number of ESTs per tissue from EST 


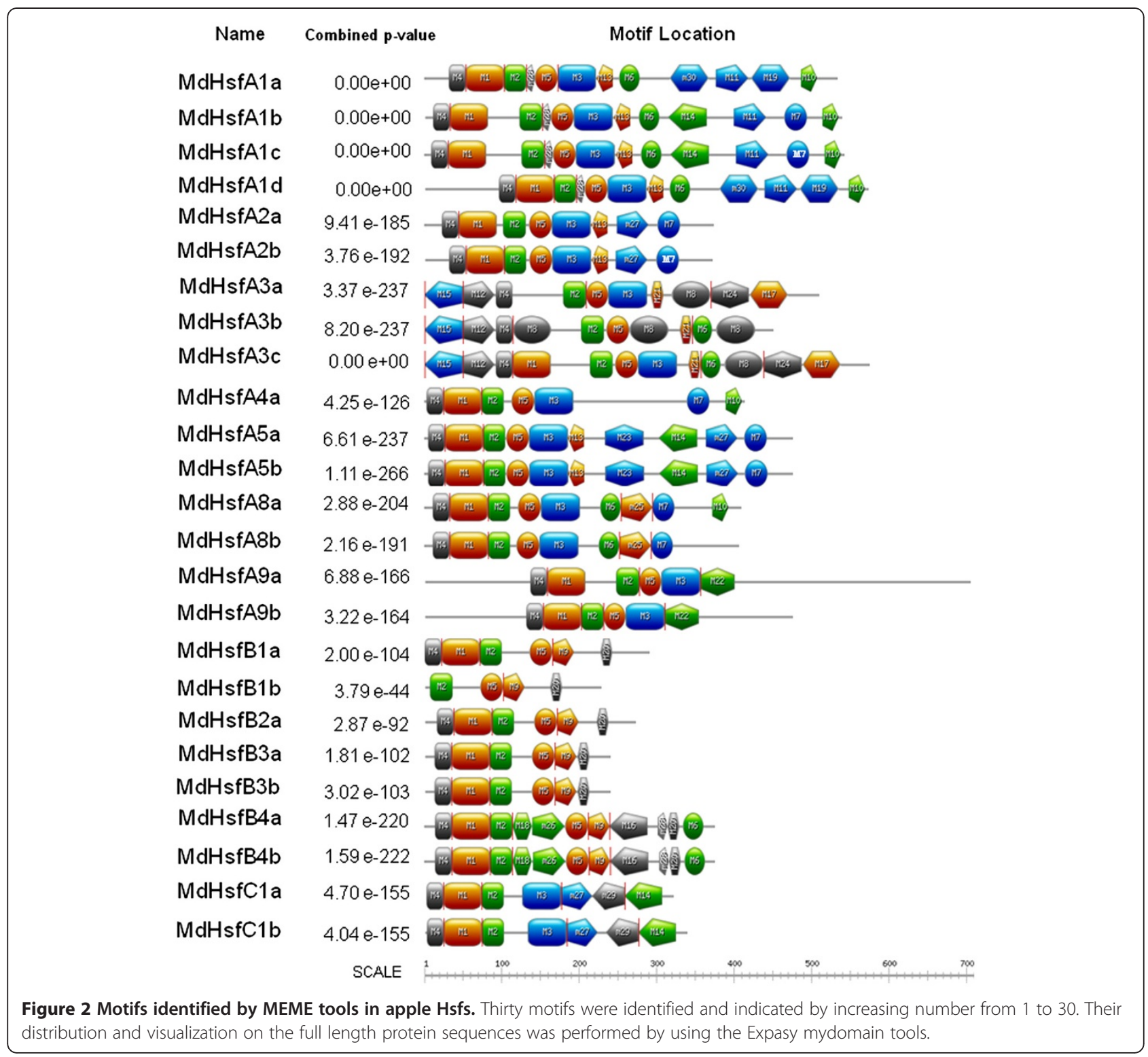

libraries [17]. This resulted in the assignment of $M d H s f s$ to nine groups on the basis of the tissue and organ types in which $M d H s f s$ were present (Table 4).

Of the group A1, MdHsfA1a and MdHsfA1d, were the most represented as their expression was detected in leaf, flower, fruit, shoot and phloem. Similarly, $M d H s f B 1 a$ and $M d H s f B 1 b$ of B class were expressed in several apple tissues. Interestingly, $M d H s f A 9 b$ was the only Hsf specific for seed, whereas $M d H s f A 9 a$ was found in leaf. Furthermore, expression restricted to only a single tissue type was observed also for other members of the $M d H s f$ family; all A3-type $M d H s f s$ were expressed in shoot and both members of the class $C$ were found in root. In addition, the analysis of digital data showed that duplicated genes located on different chromosomes had identical expression patterns (e.g. MdHsfB4a and $M d H s f B 4 b, M d H s f C 1 a$ and $M d H s f C 1 b)$.

Expression analysis of $M d H s f$ genes in apple organs under natural environmental conditions

$H s f$ genes are differentially expressed during flower and fruit development and are induced by abiotic environmental factors [7,12,13]. To investigate if $M d H s f s$ are also involved in these processes, a comprehensive analysis of their expression was performed in flowers and fruits from field-grown trees. Flowers were harvested at the stages of tight cluster, full pink and anthesis (FLS1, FLS2, FLS3) during spring at average temperatures of $23^{\circ} \mathrm{C} / 7^{\circ} \mathrm{C}$ (day/night; $\mathrm{max} / \mathrm{min}$ ), while the developing fruits were chosen at the stage of 10,15 and $20 \mathrm{~mm}$ in 
Table 3 Motif sequences identified by MEME tools in apple Hsfs

\begin{tabular}{|c|c|c|}
\hline Motif & Length & Best possible match \\
\hline 1 & 50 & TDHIISWSDANNSFWWDPPEFARDLLPKYFKHNNFSSFIRQLNTYGFRK \\
\hline 2 & 29 & VDPDRWEFANEWFQRGQKHLLCNIHRRKH \\
\hline 3 & 50 & LMQEIVRLRQQQQYTQNQLHAMNQRLQGMECRQQQMMSFLAKAMHNPGFL \\
\hline 4 & 21 & LHKTGPPPFLCKTYDMVDDPA \\
\hline 5 & 29 & YQQNPTGACVEVGKCGLWDEIERLKRDKN \\
\hline 6 & 26 & CHKYMDGQIVKYQPPMNEAAKAMLRP \\
\hline 7 & 29 & TPYTHPDIVNDIFWEQFWTARPICGNIEE \\
\hline 8 & 50 & FEQSPHYPSQVTTGKLGLDAESTAFQFVDAALDELAITQGFLETPEQEGE \\
\hline 9 & 28 & MLMSELAHMKKKCNEIIYFVANYVCMAW \\
\hline 10 & 20 & GWDKSQNMNHITEQMGHLTS \\
\hline 11 & 41 & QTDWIPELTRIQGIVPEGNVDIPNANMIGEDIGNGFYMGM \\
\hline 12 & 41 & EFEAFCSVNPLGAFDFTEKVSIPTSSMGGGGAEDWVPPQP \\
\hline 13 & 20 & HVHKNEKNRRITGYNKKRRL \\
\hline 14 & 49 & IINPDAMLITKAPTGATNTRNSSQPGYGYTNGGGGHISCEVNYPTESTP \\
\hline 15 & 50 & MSPKDESHPKSPPTSAEFDPESIGLSEFRPQVSAPLLGSQPIPSFTSPVM \\
\hline 16 & 50 & PSNSYPSSMLLCNPQPPKHNGPNGNLNQLQGYYPAAPPPNAKQNPHHIMN \\
\hline 17 & 49 & MIKQEDIWSMGFGVSAGMSTSMHELWGNPVNYDVPEMGVTGGLLDWWDI \\
\hline 18 & 26 & AQPHQVGLNHHHHHHSPLGMNGHHHH \\
\hline 19 & 50 & DGFIDPTSEVMNGSLPIDFDDISSDIEAFLKDWDDIIQNPGADEMDSTCA \\
\hline 20 & 14 & EEECKNLKLFGWWL \\
\hline 21 & 15 & VRRKFVKHQQHELSK \\
\hline 22 & 45 & QQLMQKRMIKRELDGGDLGKRRRLPPAQGIESFDEWINDSLSFDC \\
\hline 23 & 50 & FHQDFSSKLRLELSPAVSDMNLVSRSTQSSNEDGGSSTRKISEELKGAQM \\
\hline 24 & 50 & GASSMVTEDPFFKGKSVLSPQQEANPERYVSFQEDLVKDRTFPELFSPGM \\
\hline 25 & 41 & NSGSEKQPEVDAYMDGMEDFWNPDFMKMLMDEKLSPVENH \\
\hline 26 & 41 & FFPFPSRGSISPSDSDEQPNWCDSDSPPLLSPTGGINTNIN \\
\hline 27 & 40 & PRMIQEIDYSAAAELGEKAKMVMMIAFTSSTAADDDKTTT \\
\hline 28 & 11 & THVHDHQQQPP \\
\hline 29 & 41 & ISSSPEAGFEMESFNRYPTPPEVQTASDWLRQRWFVDRVRA \\
\hline 30 & 50 & CVSGVTLQEVPLTSGHGLPSVISETHSPPRVANPGTVMRSPFSDVNALVG \\
\hline
\end{tabular}

Numbers in the first column indicate the motifs represented in the Figure 2.

diameter (FUS1, FUS2, FUS3) and harvest at average temperatures of $23^{\circ} \mathrm{C} / 14^{\circ} \mathrm{C}$ (day/ night; $\max / \mathrm{min}$ ). Quantitative real-time PCR was used as the approach to monitor gene expression changes, and MdHsf transcript abundance in developing flowers/fruits was compared to that of vegetative leaf tissue $(3-5 \mathrm{~cm}$ in length). The transcriptional patterns could be analyzed only for 20 $M d H s f$ genes, since it was not possible to design specific primers discriminating MdHsfA5a-b, MdHsfA8a-b, $M d H s f B 3 a-b, M d H s f B 4 a-b$ and MdHsfC1 $a-b$ because of high sequence similarity with the corresponding isoform. Figure 4 shows the results of this analysis. Members of A1 subgroups such as MdHsfAla-d exhibited transcript accumulation in all tissues analyzed, although a higher induction was observed in flowers especially at anthesis.
Similar to the A1-subgroup, higher messenger RNA levels at anthesis were also observed for other members of A class such as MdHsfA2a-b, MdHsfA3b-c, $M d H s f A 5 a-b$ and $M d H s f A 9 a$. Interestingly, $M d H s f A 9 b$ was approximately 4-fold more strongly induced in the youngest flowers than at the later stages. A broad variability of expression patterns in flowers and fruits was instead observed for the MdHsf members belonging to the B class. $M d H s f B 1 a$ displayed a similar trend as a large part of the A members with higher expression at anthesis. On the contrary, its duplicated gene $M d H s f B 1 b$ did not show any remarkable transcript changes between the different flower and fruit stages. $M d H s f B 2 a$ showed strong upregulation in $20 \mathrm{~mm}$ fruit where it was 30 -fold higher than in leaf tissue. The B4-type gene MdHsfB4a-b exhibited a 


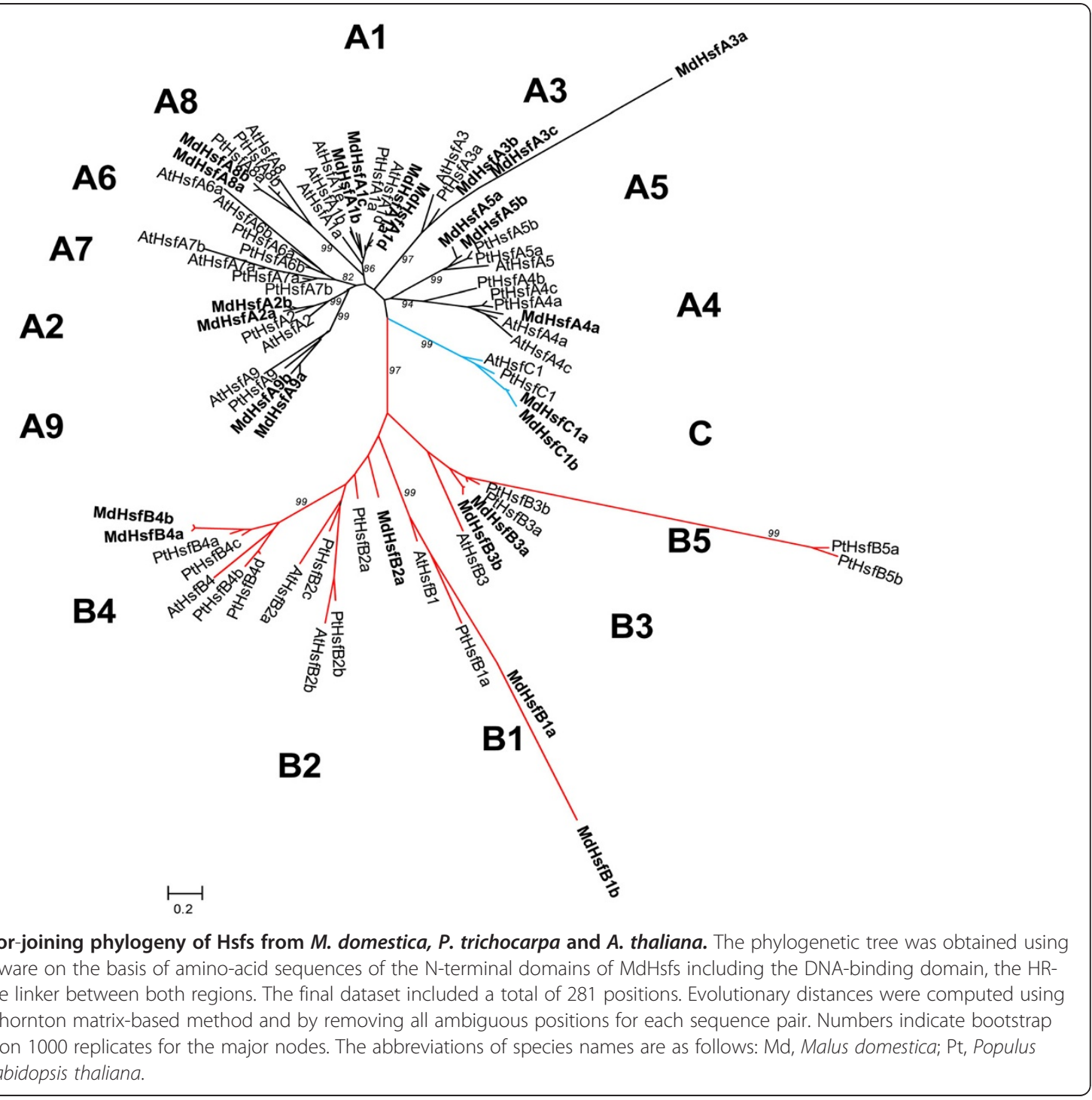

lower expression in flower/fruit than in leaf. Low transcript abundances in fruit as compared to flower or leaf were also observed for $M d H s f C l a-b$.

To further characterize the expression of Hsf family genes in apple, the quantitative real-time PCR analysis was extended to leaf samples harvested from field-grown trees exposed to naturally increased temperatures. Leaf samples were taken during the summer period, at two different temperature ranges: at $26^{\circ} \mathrm{C} / 12^{\circ} \mathrm{C}$ (day/night; $\mathrm{max} / \mathrm{min}$ ) on 30th July 2011, which were used as reference, and at high temperature average of $32^{\circ} \mathrm{C} / 17^{\circ} \mathrm{C}$ (day/night; $\mathrm{max} / \mathrm{min}$ ) on the 21st August 2011 (Additional file 1: Figure S1).

The transcriptional analyses revealed that in leaf most of the MdHsfs genes were responsive to the increased temperatures (Figure 5). Twelve of these responsive genes showed transcript accumulation significantly higher than the reference sample, while only $M d H s f A 9 b$ and $M d H s f B 4 a-b$ were strongly down-regulated in response to the increased temperatures. A 4-fold or higher increase of expression levels in response to high temperatures was observed for MdHsfA2a-b, MdHsfA3b-c, $M d H s f B 1 a, M d H s f B 2 a, M d H s f B 3 a-b$ and MdHsfC1a-b, and only slightly higher in the stressed leaves than in the reference onces for MdHsfA4a, MdHsfA5a-b and $M d H s f A 8 a-b$. Furthermore, all subgroup A1 members such as MdHsfA1a-d did not show any significant transcriptional changes in response to the high temperatures compared to the reference conditions.

\section{Discussion}

In plants, members of the Hsf family have been described as key regulators in molecular and cellular responses to stress conditions [1,7]. Furthermore, data from tomato and Arabidopsis have shown that the Hsfs are important components involved in developmental signalling $[13,14]$. Both size and composition of the Hsf family have been 
Table 4 Digital expression of $M d o H s f$ genes

\begin{tabular}{|c|c|c|c|c|c|c|c|c|c|}
\hline \multicolumn{10}{|c|}{ Tissue and organ type (DFCI Apple Gene Index) } \\
\hline Gene name & Leaf & Root & Flower & Fruit & Shoot & Phloem & Xylem & Seed & Bud \\
\hline MdHsfAla & + & & + & + & + & + & & & \\
\hline MdHsfA1b & & & & + & & & & & \\
\hline MdHsfAlc & & & & + & & & & & \\
\hline MdHsfAld & + & & + & + & + & + & & & \\
\hline MdHsfAZa & & + & + & & + & & & & \\
\hline MdHsfA2b & & + & & & & & & & \\
\hline MdHsfA3a & & & & & + & & & & \\
\hline MdHsfA3b & & & & & + & & & & \\
\hline MdHSfA3C & & & & & + & & & & \\
\hline MdHsfA4a & & + & + & & & & & & \\
\hline MdHsfA5a & & + & + & + & & & & & \\
\hline MdHsfA5b & & + & + & + & & & & & \\
\hline MdHsfABa & & & & + & & & & & \\
\hline MdHsfABb & & & & + & & & & & \\
\hline MdHsfA9a & + & & & & & & & & \\
\hline MdHsfA9b & & & & & & & & + & \\
\hline MdHsfBla & + & + & & + & & + & + & & \\
\hline$M d H s f B 1 b$ & + & + & & + & & + & + & & \\
\hline$M d H s f B 2 a$ & & & & & & + & & & \\
\hline$M d H s f B 3 a$ & & & + & & & & & & \\
\hline$M d H s f B 3 b$ & & & + & & & & & & \\
\hline$M d H s f B 4 a$ & & & & & & & & & + \\
\hline$M d H s f B 4 b$ & & & & & & & & & + \\
\hline $\mathrm{MdHsfCla}$ & & + & & & & & & & \\
\hline MdHsfClb & & + & & & & & & & \\
\hline
\end{tabular}

+: Expressed; blank: not expressed.

analyzed and characterized in different plant species [1]. The present study investigates for the first time this gene family in the economically relevant domesticated apple and shows that its genome contains 25 full length $H s f$ genes. This number is similar to that of Populus trichocarpa for which 28 loci encoding Hsf proteins were found [10]. Velasco et al. [2010] have shown that genome wide duplications had occurred in apple causing the expansion of several gene classes. Indeed, it was found that the enlargement of the $M d H s f$ family is in particular originated from segmental duplications between different chromosomes. This situation is similar in maize and in Populus, in which segmental $H s f$ gene duplications were more prevalent than those of tandem duplications [9,10]. Gene duplications have an important role not only in the genomic rearrangement and expansion but also in diversification of gene function. In particular, genes encoding for nucleic acid binding proteins, among which transcription factors, originated mostly by segmental duplication. In contrast, membrane proteins and proteins involved in the stress response are encoded by genes mainly duplicated in tandem $[18,19]$. Therefore, the prevalence of segmental duplication events in $M d H s f$ expansion may be associated to the fact that these genes act as transcriptional regulators.

Malus, Arabidopsis and Populus belong to the Rosid lineage and they are grouped in two distinct clades, namely Fabids (Malus and Populus) and Malvids (Arabidopsis) [20]. It was observed in the present study that the majority of the MdHsfs had a closer phylogenetic relationship to the PtHsfs than to the AtHsfs. This may be attributable to the fact that Malus and Populus belong to the same Fabids clade, and as they are both trees may have adapted to prolonged and repeated environmental constraints, unlike Arabidopsis.

Functional diversification of multifamily duplicated genes has been observed in trees. For example, the family of the glutathione S-transferase in Populus has a clear divergence in expression patterns in response to different stress treatments [21]. Therefore the presence of many duplicated $H s f$ genes in the apple genome may be 


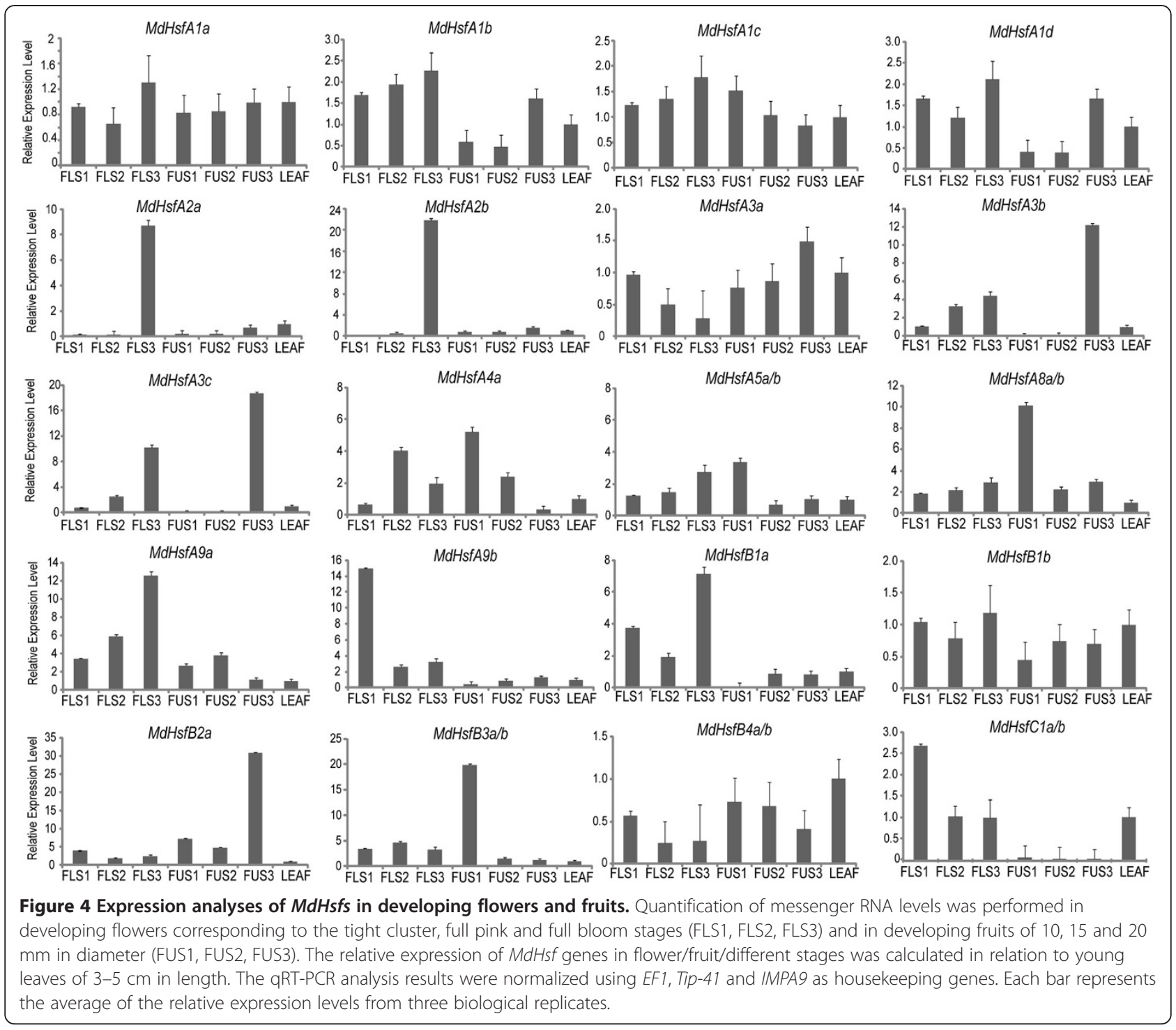

related to the fact that a sub-functionalization has taken place especially to cope with prolonged and specific stress conditions.

$M d H s f$ genes were found to be expressed in several apple tissues. In particular, members belonging to the A1 and B1 subclasses, such as MdHsfA1a, MdHsfA1d, $M d H s f B 1 a, M d H s f B 1 b$, were constitutively expressed in different tissues. A similar situation was found in other plants like Arabidopsis where A1-type $\mathrm{Hsfs}$ were involved in house-keeping processes under normal conditions, being ready for the fast activation of other Hsfs genes following stress treatment [22,23]. Furthermore, expression data from flower and fruit tissues indicated that some duplicated gene pairs, e.g. MdHsfA9a and $M d H s f A 9 b$, exhibited differences in their expression levels. This suggests that they may be subjected to a different regulation in apple tissue $[1,7]$.
In contrast, the expression of $M d H s f A 2 a$ and $M d H s f A 2 b$ was mainly detected in full bloom flowers. AtHsfA9 and LeHsfA2a (Le, Lycopersicon esculentum) were found expressed in seed and developing pollen grains $[13,14,24]$. It was shown that the presence of these Hsfs during plant development is important for heat shock protein activation. This suggests that MdHsfA2a and MdHsfA2b may be important during pollination and fertilization, which occurs at anthesis.

Effects of heat stress (HS) on Hsf gene expression has been examined in several plant species, but no data are available about $H s f$ expression in trees exposed to naturally increased temperatures. Under laboratory settings, it was shown that AtHsfAla and AtHsfA1b regulate the early response to HS in Arabidopsis $[22,25]$. AtHsfA2 is rapidly induced by HS, and it is involved in enhancing and maintaining of HS-response 


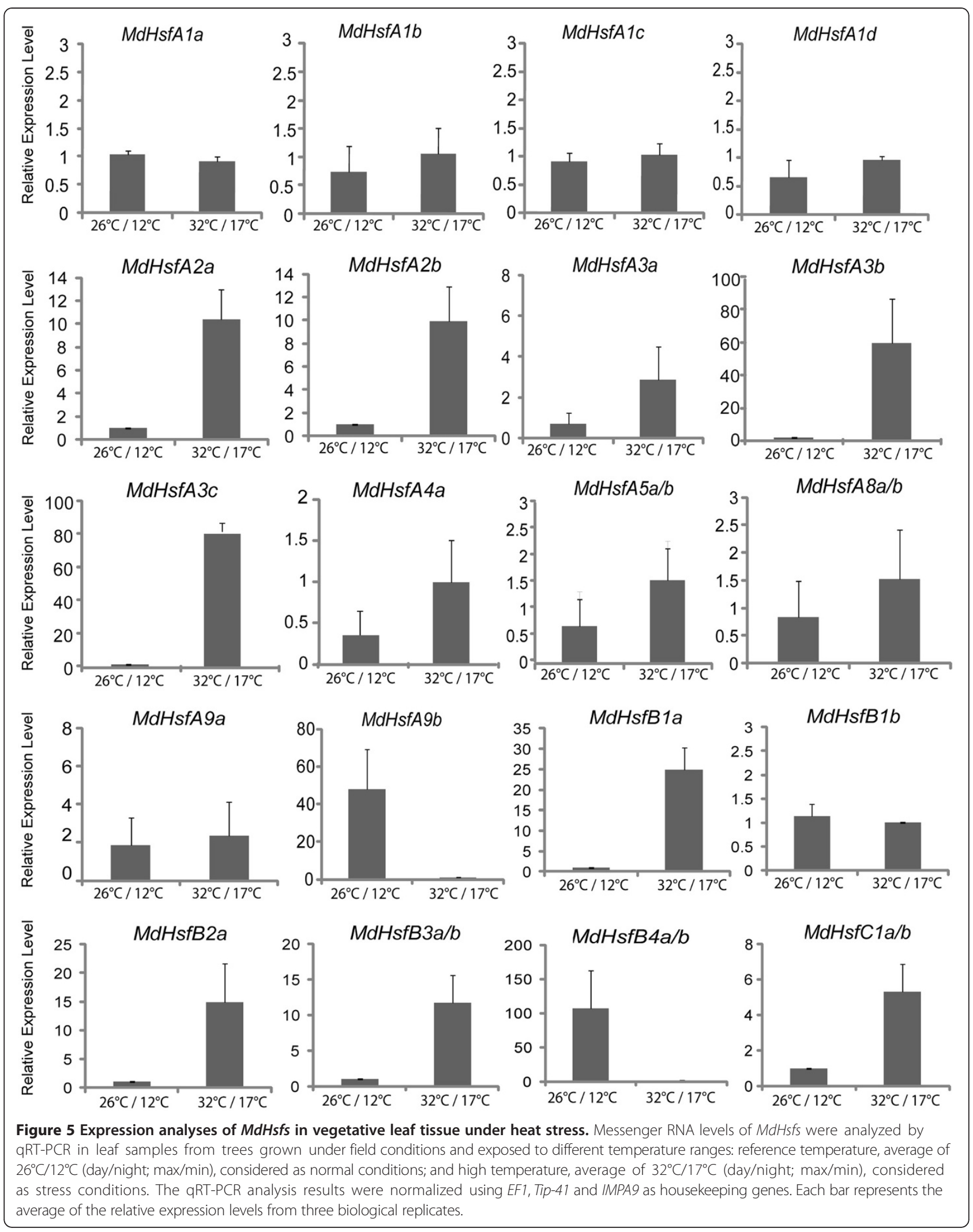


when plants are exposed to prolonged or repeated cycles of HS $[26,27]$. Similarly to AtHsfA2, AtHsfA3 is involved in thermo-tolerance mechanisms $[7,28,29]$. The A1-type $M d H s f s$ were expressed at the same level also in leaves from plants growing in field and exposed to different temperature conditions. MdHsfA2a-b, $M d H s f A 3 b-c$ were instead strongly induced. This may suggest that these types of MdHsfs could be involved in maintaining the stress response when apple trees are exposed to prolonged periods of high temperature conditions.

In contrast to class A Hsfs, genes assigned to the B and $\mathrm{C}$ classes have so far not been fully characterized. Members of the $B$ class were shown to act mainly as repressors of the expression of HS inducible genes $[30,31]$. Some of them form a complex with Hsf A-types to maintain housekeeping gene expression during HS regimes [32]. Therefore, the strong transcriptional activation in apple may indicate that some of them may have a role in the response to the high temperatures also in this species. For the majority of $M d H s f s$, increased messenger RNA levels were observed under naturally increased temperatures. However, MdHsfA9b and $M d H s f B 4 a-b$ were the only $H s f$ genes showing low transcript abundance. Although proteomic data are not available for all $M d H s f s$ genes, their activation or repression may suggest that these transcripts could have a high hierarchy of molecular events induced by the high temperatures.

\section{Conclusions}

The complexity of the Hsf family has been object of many investigations in different plant species. Here, 25 full length $H s f s$ genes were identified in the apple genome. Based on structural characteristics of the proteins and on the comparison with homologues from other species, the $25 \mathrm{MdHsfs}$ were grouped in three different classes. Segmental and tandem duplications were examined and contributed to the expansion of the Hsf family in the apple genome. The expression profiles in flowers/ fruits at different developmental stages as well as in leaves exposed to naturally increased temperature indicated that $M d H s f s$ may play a role in different aspects of apple growth/development.

Malus domestica represents an economically important woody plant whose genome has been fully sequenced and whose commercial value is due to fruit production in the field. Therefore, understanding the role of protective genes as the $H s f s$ during development and under stress conditions is important. The results of this research will be undoubtedly useful for future gene cloning and functional studies and, in turn, for producing apple cultivars with improved genetic traits.

\section{Methods}

Identification and classification of Hsfs in Malus domestica The recently sequenced apple genome was investigated for putative genes encoding for MdHsfs (Md: Malus domestica) based on BLASTN and BLASTP in NCBI and TIGR-Apple databases [11,15]. Physical localization of all candidate $M d H s f s$ was analyzed in order to reject redundant sequences with the same chromosome location. In order to identify signature domains, the MdHsf sequences were compared to the Hsf proteins of Arabidopsis and tomato by amino acid sequence alignment using ClustalW (version 1.83). Presence of DBD domains and coiled-coil structures were checked by SMART and MARCOIL programs [33,34]. In addition, identification of putative domain motifs in the full-length amino acid sequences of the MdHsfs was also performed by MEME tools [35]. Visualization of the Meme motifs in the MdHsfs was performed by using Expasy tools (http:// prosite.expasy.org/mydomains). MdHsf names were assigned on the basis of the original nomenclature as worked out for the Arabidopsis thaliana Hsf family, and later applied to other plant Hsf families [2,7]. Classification into three different groups $\mathrm{A}, \mathrm{B}$ and $\mathrm{C}$ was based on the information of oligomerization domains [2].

\section{Phylogenetic analysis and gene duplication of $\mathrm{MdHsfs}$}

Gene duplications in the apple genome were analyzed by testing the similarity of all MdHsf genes using ClustalW. A gene duplication was defined according to the following criteria: (1) the length of the sequence alignment covered $\geq 80 \%$ of the longest gene, and (2) the similarity of the aligned gene regions was $\geq 80 \%[36,37]$. Data were then plotted using Circos software [38].

To understand the evolutionary relationships of the MdHsf proteins, a phylogenetic tree was constructed. The $\mathrm{N}$-terminal Hsf protein sequences containing the DBD and HR-A/B regions from Malus domestica, Arabidopsis thaliana and Populus trichocarpa $[7,10]$ were aligned using ClustalW. A phylogenetic tree was constructed using the Neighbor Joining (NJ) method in MEGA (version 5.0) [39]. Based on the results of the model selection analysis, the Jones-Taylor-Thornton matrix-based method was used to compute evolutionary distances [40]. The rate variation among sites was modeled with a gamma distribution (shape parameter = 0.67). Bootstrap analysis was conducted with 1000 replicates to assess statistical support for each node.

\section{Digital and EST expression analysis}

The analysis of $M d H s f s$ expression profiles was investigated at the transcriptional level. $M d H s f s$ expression patterns were searched with the BLAST program in TIGR-Apple EST libraries [17] using the following 
parameters: maximum identity $>95 \%$, length $>200 \mathrm{bp}$ and E-value $<10^{-10}$.

\section{Plant material}

Experiments were carried out in 2011 on 18-year-old apple trees (cultivar 'Golden Delicious' on M9 rootstock) trained with standard horticultural practices at the experimental farm of the Research Centre for Agriculture and Forestry Laimburg (South Tyrol, Italy). Samples were taken from 24 homogeneous trees grouped in 3 biological replicates each containing 8 trees distributed in the same block of the orchard. Tissue samples were collected between April and August 2011 from trees grown under field environmental conditions and exposed to natural variations of temperature and solar radiation. Temperature data are reported in the Additional file 1. Young leaves $(3-5 \mathrm{~cm}$ in length) as well as developing flowers corresponding to the tight cluster (FLS1), pink (FLS2) and full bloom (anthesis, FLS3) stages were harvested from the plants during spring period and under max-minimum temperature average in the range of $23^{\circ} \mathrm{C} /$ $7^{\circ} \mathrm{C}$ (day/night; $\max / \mathrm{min}$ ). From the same trees developing fruits of $10 \mathrm{~mm}$ (FUS1), $15 \mathrm{~mm}$ (FUS2) and $20 \mathrm{~mm}$ (FUS3) in length were also collected under max-minimum temperatures of $23^{\circ} \mathrm{C} / 14^{\circ} \mathrm{C}$ (day/night; $\max / \mathrm{min}$ ). For testing $H s f s$ gene expression under naturally increased temperature conditions, leaf samples were taken during the summer period, at two different temperature ranges: at $26^{\circ} \mathrm{C} / 12^{\circ} \mathrm{C}$ (day/night; $\max / \mathrm{min}$ ) on 30th July, 2011, which were used as reference, and at high temperature average of $32^{\circ} \mathrm{C} / 17^{\circ} \mathrm{C}$ (day/night; $\max / \mathrm{min}$ ) on the 21st August, 2011 (Additional file 1: Figure S1). All samples used in gene expression analyses were harvested at midday $(12: 00 \mathrm{am})$ and were positioned around $1.60 \mathrm{~m}$ in height from the soil.

\section{RNA isolation and quantitative real-time PCR (qRT-PCR) analyses}

Total RNA was isolated from apple tissues with the hot phenol method [41]. RNA quantity was measured using a NanoDrop ND-1000 spectrophotometer, and its quality was checked by agarose gel electrophoresis. For reverse transcription, total RNA was incubated with RNase-free DNase (RQ1; Promega, Madison, WI), and 1 $\mu \mathrm{g}$ was used for reverse transcription according to the manufacturer's instructions (Superscript Vilo cDNA Synthesis kit; Invitrogen).

The qRT-PCR analyses were carried out on a 7500 Fast Real-time PCR System (Applied Biosystems) with the ROX Reference Dye. Each reaction contained $12.5 \mu \mathrm{l}$ SYBR GreenER qPCR SuperMix Universal (Invitrogen), $20 \mathrm{ng}$ of cDNA and $400 \mathrm{nM}$ of each specific primer. The qRT-PCRs were performed using a controlled temperature program starting with $10 \mathrm{~min}$ at $95^{\circ} \mathrm{C}$, followed by 40 cycles of $15 \mathrm{~s}$ at $95^{\circ} \mathrm{C}$ and $60 \mathrm{~s}$ at $60^{\circ} \mathrm{C}$. To verify the presence of a specific product, the melting temperature of the amplified products was determined. In addition, each PCR mixture was analyzed on a $2 \%$ agarose/ethidium bromide stained gel to verify the size of the amplified DNA fragment. The primers used for the qRT-PCRs were designed using Quantprime software and are reported in the Additional file 2 [42]. The qRT-PCRs were performed in duplicated technical reactions and repeated on three independent biological replicates. Relative mRNA levels of the target genes were calculated based on Vandesompele et al. [2002] [43]. The genes encoding for elongation factor 1 alpha subunit (eF-1 alpha; accession number AJ223969.1), Importin alpha Isoform9 (IMPA-9; accession number CN909679) and Tip-41 like protein (Tip-41 CN941833) were used as references in the qRT-PCR analyses.

\section{Additional files}

Additional file 1: Figure S1. Average temperature in the orchard where apple trees were sampled during the 2011 growing season. The data show temperature ranges obtained from a meteorological station located in the apple orchard and positioned around $2 \mathrm{~m}$ in height. Each point represents the average calculated on the basis of data from seven days.

Additional file 2: Primer sequences used for quantitative real-time PCR analyses.

\section{Abbreviations}

Hsf: Heat shock transcriptional factor; HR-A/B: Adjacent bipartite oligomerization domain; AHA: Activator motif; CTAD: C-terminal activation domain; DBD: DNA-binding domain; HS: Heat stress; HSE: Heat shock element; NES: Nuclear export signal; NLS: Nuclear localization signal; qRTPCR: Quantitative reverse transcription real-time PCR.

\section{Competing interests}

The authors declare that they have no competing interests.

\section{Authors' contributions}

This study was designed by FG, SB and CM. Bioinformatics analyses were performed by $F G$, expression analyses by $F G$ and $G G$. FG and $G G$ assisted to tissue sampling. FG, GG, SB and CM contribute to manuscript preparation and revision. All authors have read and approved the final manuscript.

\section{Acknowledgements}

The authors wish to thank Nunzio D'Agostino for suggestions during manuscript preparation and for his useful contribution in drawing the Circos Figure 1. Christine Kerschbamer is very thanked for the technical assistance, Philipp Brunner to assist with apple growth and Alberto Storti for his kind support during this research.

The authors are grateful to the Foundation for Research and Innovation of the Autonomous Province of Bozen/Bolzano for covering the Open Access publication costs.

This work was partially funded by the Autonomous Province of Bozen/ Bolzano, Italy (Departments 31 and 33). The South Tyrolean Fruit Growers' Co-operatives, in particularly VOG and VIP, are acknowledged for cofinancing the Strategic Project on Apple Proliferation - APPL.

Received: 13 June 2012 Accepted: 8 November 2012

Published: 20 November 2012 


\section{References}

1. von Koskull-Döring P, Scharf KD, Nover L: The diversity of plant heat stress transcription factors. Trends Plant Sci 2007, 12:452-457.

2. Nover L, Bharti K, Döring P, Mishra SK, Ganguli A, Scharf KD: Arabidopsis and the heat stress transcription factor world: how many heat stress transcription factors do we need? Cell Stress Chaperone 2001, 6:177-189.

3. Döring P, Treuter E, Kistner C, Lyck R, Chen A, Nover L: The role of AHA motifs in the activator function of tomato heat stress transcription factors HsfA1a and HsfA2. Plant Cell 2000, 12:265-278.

4. Morimoto RI: Regulation of the heat shock transcriptional response: crosstalk between a family of heat shock factors, molecular chaperones and negative regulators. Genes Dev 1998, 12:3788-3796.

5. Pirkkala L, Nykanen P, Sistonen L: Roles of the heat shock transcription factors in regulation of the heat shock response and beyond. FASEB $J$ 2001, 15:1118-1131.

6. Baniwal SK, Bharti K, Chan KY, Fauth M, Ganguli A, Kotak S, Mishra SK, Nover L, Port M, Scharf KD, Tripp J, Weber C, Zielinski D, Döring P: Heat stress response in plants: a complex game with chaperones and more than twenty heat stress transcription factors. J Biosci 2004, 29:471-487.

7. Scharf KD, Berberich T, Ebersberger I, Nover L: The plant heat stress transcription factor (Hsf) family: Structure, function and evolution. Biochim Biophys Acta 2012, 1819(2):104-119.

8. Chauhan H, Khurana N, Agarwal P, Khurana P: Heat shock factors in rice (Oryza sativa L.): genome-wide expression analysis during reproductive development and abiotic stress. Mol Genet Genomics 2011, 286(2):171-187.

9. Lin YX, Jiang HY, Chu ZX, Tang XL, Zhu SW, Cheng BJ: Genome-wide identification, classification and analysis of heat shock transcription factor family in maize. BMC Genomics 2011, 12:76.

10. Wang F, Dong Q, Jiang H, Zhu S, Chen B, Xiang Y: Genome-wide analysis of the heat shock transcription factors in Populus trichocarpa and Medicago truncatula. Mol Biol Rep 2012, 39(2):1877-1886.

11. Velasco R, Zharkikh A, Affourtit J, Dhingra A, Cestaro A, Kalyanaraman A, Fontana P, Bhatnagar SK, Troggio M, Pruss D, Salvi S, Pindo M, Baldi P, Castelletti S, Cavaiuolo M, Coppola G, Costa F, Cova V, Dal Ri A Goremykin V, Komjanc M, Longhi S, Magnago P, Malacarne G, Malnoy M, Micheletti D, Moretto M, Perazzolli M, Si-Ammour A, Vezzulli S, et al: The genome of the domesticated apple (Malus domestica Borkh). Nat Genet 2010, 42(10):833-839.

12. Frank G, Pressman E, Ophir R, Althan L, Shaked R, Freedman M, Shen S, Firon N: Transcriptional profiling of maturing tomato (Solanum lycopersicum L.) microspores reveals the involvement of heat shock proteins, ROS scavengers, hormones, and sugars in the heat stress response. J Exp Bot 2009, 60(13):3891-3908.

13. Giorno F, Wolters-Arts M, Grillo S, Scharf KD, Vriezen WH, Mariani C: Developmental and heat stress-regulated expression of $\mathrm{HsfA} 2$ and small heat shock proteins in tomato anthers. J Exp Bot 2010, 61(2):453-462

14. Kotak S, Vierling E, Bäumlein $H$, von Koskull-Döring P: A novel transcriptional cascade regulating expression of heat stress proteins during seed development of Arabidopsis. Plant Cell 2007, 19:182-195.

15. GDR: http://www.rosaceae.org/projects/apple_genome.

16. Vision TJ, Brown DG, Tanksley SD: The origins of genomic duplications in Arabidopsis. Science 2000, 290(5499):2114-2117.

17. TIGR: http://www.tigr.org/.

18. Hanada K, Zou C, Lehti-Shiu MD, Shinozaki K, Shiu SH: Importance of lineage-specific expansion of plant tandem duplicates in the adaptive response to environmental stimuli. Plant Physiol 2008, 148(2):993-1003.

19. Rizzon C, Ponger L, Gaut BS: Striking similarities in the genomic distribution of tandemly arrayed genes in Arabidopsis and rice. PLOS Comput Biol 2006, 2:e115

20. Judd WS, Olmstead RG: A survey of tricolpate (eudicot) phylogenetic relationships. Am J Bot 2004, 91(10):1627-1644.

21. Lan T, Yang ZL, Yang X, Liu YJ, Wang XR, Zeng QY: Extensive functional diversification of the Populus glutathione S-transferase supergene family. Plant Cell 2009, 21(12):3749-3766.

22. Busch W, Wunderlich M, Schöffl F: Identification of novel heat shock factor-dependent genes and biochemical pathways in Arabidopsis thaliana. Plant J 2005, 41(1):1-14

23. Mishra SK, Tripp J, Winkelhaus S, Tschiersch B, Theres K, Nover L, Scharf KD: In the complex family of heat stress transcription factors, HsfA1 has a unique role as master regulator of thermotolerance in tomato. Genes Dev 2002, 16(12):1555-1567.

24. Almoguera C, Rojas A, Díaz-Martín J, Prieto-Dapena P, Carranco R, Jordano J: A seed-specific heat-shock transcription factor involved in developmental regulation during embryogenesis in sunflower. J Biol Chem 2002, 277(46):43866-43872.

25. Lohmann C, Eggers-Schumacher G, Wunderlich M, Schöffl F: Two different heat shock transcription factors regulate immediate early expression of stress genes in Arabidopsis. Mol Genet Genomics 2004, 271(3):376.

26. Charng YY, Liu HC, Liu NY, Chi WT, Wang CN, Chang SH, Wang TT: A heat-inducible transcription factor, HsfA2, is required for extension of acquired thermotolerance in Arabidopsis. Plant Physiol 2007, 143(1):251-262.

27. Meiri D, Breiman A: Arabidopsis ROF1 (FKBP62) modulates thermotolerance by interacting with HSP90.1 and affecting the accumulation of HsfA2-regulated sHSPs. Plant J 2009, 59(3):387-399.

28. Schramm F, Larkindale J, Kiehlmann E, Ganguli A, Englich G, Vierling E, von Koskull-Döring P: A cascade of transcription factor DREB2A and heat stress transcription factor $\mathrm{HsfA} 3$ regulates the heat stress response of Arabidopsis. Plant J 2008, 53(2):264-274.

29. Chen H, Hwang JE, Lim CJ, Kim DY, Lee SY, Lim CO: Arabidopsis DREB2C functions as a transcriptional activator of $\mathrm{HsfA} 3$ during the heat stress response. Biochem Biophys Res Commun 2010, 401(2):238-244.

30. Czarnecka-Verner E, Yuan CX, Scharf KD, Englich G, Gurley WB: Plants contain a novel multi-member class of heat shock factors without transcriptional activator potential. Plant Mol Biol 2000, 43:459-471.

31. Ikeda M, Mitsuda N, Ohme-Takagi M: Arabidopsis HsfB1 and HsfB2b act as repressors of the expression of heat-inducible Hsfs but positively regulate the acquired thermotolerance. Plant Physiol 2011, 157(3):1243-1254.

32. Bharti K, Von Koskull-Döring P, Bharti S, Kumar P, Tintschl-Körbitzer A, Treuter $\mathrm{E}$, Nover $\mathrm{L}$ : Tomato heat stress transcription factor $\mathrm{HsfB} 1$ represents a novel type of general transcription coactivator with a histone-like motif interacting with the plant CREB binding protein ortholog HAC1. Plant Cell 2004, 16(6):1521-1535.

33. SMART: http://smart.embl/heidelberg.de/.

34. MARCOIL: http://toolkit.tuebingen.mpg.de/marcoil.

35. Bailey TL, Williams N, Misleh C, Li WW: MEME: discovering and analyzing DNA and protein sequence motifs. Nucleic Acids Res 2006, 34:W369-W373.

36. Yang S, Zhang X, Yue JX, Tian D, Chen JQ: Recent duplications dominate NBS-encoding gene expansion in two woody species. Mol Genet Genomics 2008, 280:187-198.

37. Gu Z, Cavalcanti A, Chen FC, Bouman P, Li WH: Extent of gene duplication in the genomes of Drosophila, nematode, and yeast. Mol Biol Evol 2002, 19:256-262.

38. Krzywinski M, Schein J, Birol I, Connors J, Gascoyne R, Horsman D, Jones SJ, Marra MA: Circos: an information aesthetic for comparative genomics. Genome Res 2009, 19:1639-1645.

39. Tamura K, Dudley J, Nei M, Kumar S: MEGA4: Molecular Evolutionary Genetics Analysis (MEGA) software version 4.0. Mol Biol Evol 2007, 24:1596-1599.

40. Jones DT, Taylor WR, Thornton JM: The rapid generation of mutation data matrices from protein sequences. Comput Appl Biosci 1992, 8:275-282.

41. Gambino G, Perrone I, Gribaudo I: A Rapid and effective method for RNA extraction from different tissues of grapevine and other woody plants. Phytochem Anal 2008, 19(6):520-525.

42. Arvidsson S, Kwasniewski M, Riaño-Pachón DM, Mueller-Roeber B: QuantPrime-a flexible tool for reliable high-throughput primer design for quantitative PCR. BMC Bioinforma 2008, 9:465.

43. Vandesompele J, De Preter K, Pattyn F, Poppe B, Van Roy N, De Paepe A Speleman F: Accurate normalization of real-time quantitative RT-PCR data by geometric averaging of multiple internal control genes. Genome Biology 2002, 3(7):1-11. 3 RESEARCH0034.

doi:10.1186/1471-2164-13-639

Cite this article as: Giorno et al:: Heat shock transcriptional factors in Malus domestica: identification, classification and expression analysis. BMC Genomics 2012 13:639. 